\title{
Bioinformatics Knowledge Transfer Programme [KTP] for Human and Capital Development in South Africa
}

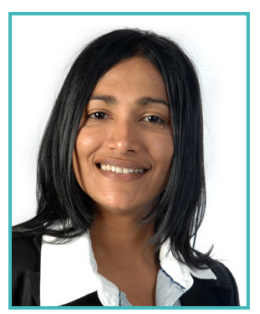

\section{Judit Kumuthini ${ }^{\bowtie}$, Emile Chimusa, Velaphi Masilela}

Centre for Proteomics and Genomic Research (CPGR), South Africa Kumuthini J (2014) EMBnet.journal 20(Suppl A), e791. htttp://dx.doi.org/10.14806/ej. 20.A.791.

A shortage of practical bioinformatics skills and expertise hinders scientific research and sustainable growth in Africa. This largely owes to underdeveloped infrastructures coupled with rare and depleted expertise that can facilitate skills transfer, including slow internet services, limited access and availability of life science research apparatus, and few organised activities that support knowledge expansion. Further compounding the problem is that individuals with some bioinformatics knowledge rarely collaborate with other experts on the African continent and rather look to international collaborative opportunities. This in turn decreases knowledge sharing and capacity development in Africa. The KTP aims to address this problem to develop and advance bioinformatics skill levels in Africa. KTP functions by acquiring the services of global experts to provide expertise and guidance to local research projects. Through this framework, KTP aims to promote knowledge exchange, grow local capacity, and strengthen the quality of local research data with the help of experienced and accredited bioinformaticians. KTP participants will work with an expert using a 'hands-on' approach to ensure that knowledge transfer occurs in a relevant environment using applicable examples. Trainees benefit from reduced training costs, exposure to a professional biotech environment and access to bespoke bioinformatics support. Experts gain exposure and experience in an African context, are afforded an opportunity to publish or patent research findings, and establish long-term collaborative relationships. The KTP recognises that input from global experts will help to ensure sustainable bioinformatics capacity development in Africa and contribute towards the identification of solutions to African problems. 